\title{
A Stereo Electronic Nose for a Mobile Inspection Robot
}

\author{
Achim Lilienthal $^{1}$, Tom Duckett ${ }^{2}$ \\ ${ }^{1}$ University of Tübingen, WSI, D-72076 Tübingen, Germany \\ lilien@informatik.uni-tuebingen.de \\ 2 Department of Technology, AASS, Örebro University, S-70182 Örebro, Sweden \\ tdt@tech.oru.se
}

\begin{abstract}
This paper describes the design of a gas-sensitive system ("mobile nose") that is suitable for use on a mobile robot. The stereo architecture comprises two equivalent sets of gas sensors mounted inside separated ventilated tubes (or "nostrils"). To characterise the dynamic response, the whole system is modelled as a first-order sensor. The corresponding parameters, including the response and recovery time, can be obtained by fitting this model to the values recorded during a simple experiment described in this paper. Our experiments confirmed the suitability of the applied model and permitted a quantitative comparison of different set-ups. It is shown that using suction fans lowers the recovery time of the metal oxide gas sensors by a factor of two, while a solid separation between the tubes (a "septum") is necessary to maintain the sensitivity of the mobile nose to concentration gradients.
\end{abstract}

\section{Introduction}

Automatic sensing of gas distributions could be very useful for a number of industrial applications. However, while individual gas sensors can be relatively cheap, they can only cover a small area. To cover larger scale environments such as warehouses and factories with a fixed installation of sensors, an arbitrarily large number of sensors would be required, resulting in very high set-up costs. As an alternative solution, the design of a gas-sensitive system is presented (the "Mark III mobile nose") that can be used on mobile inspection robots.

One possible application of this mobile nose would be an "electronic watchman" that can detect, localise and identify odours caused by leaking solvents, hazardous gases, fires, etc. However, before such an application can be realized, there are a number of practical problems that must be addressed. For example, the metal oxide sensors used in this research are subject to a long response time and an even longer recovery time. Also, the spread of gases in typi- cal indoor environments tends to be dominated by turbulence rather than diffusion, resulting in very noisy and unpredictable sensor signals. These effects could be lessened by temporal averaging in a static sensor system, but must be minimised by some other method if a mobile system is to cover the environment at reasonable speed.

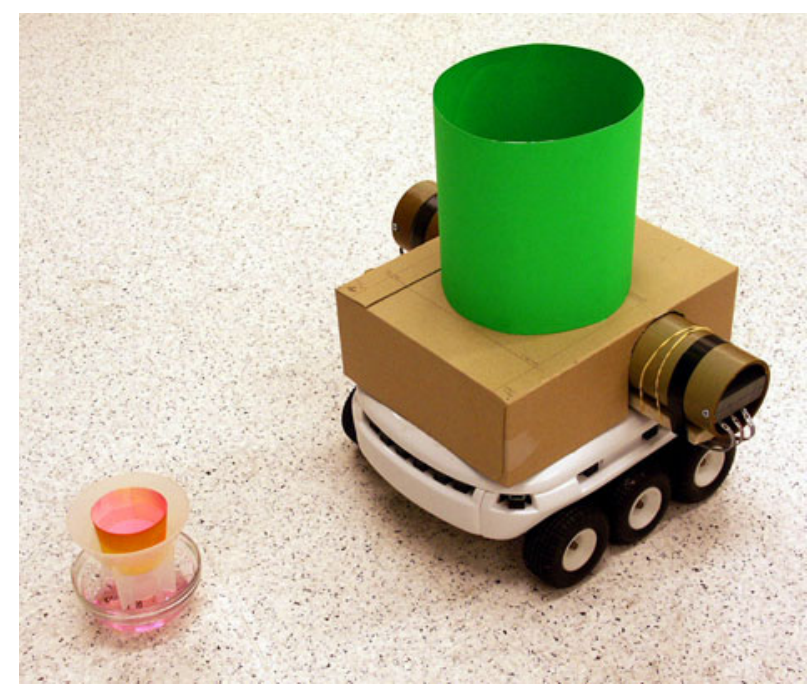

Figure 1: The Mark III mobile nose on a Koala robot facing a gas source. Two sets of 3 metal oxide gas sensors (one set is visible) were mounted inside the two suction tubes at the rear of the robot.

A mobile nose was developed to solve these problems on a K-team Koala robot (see Fig. 1) according to the following design decisions. First, it was decided to use metal oxide sensors with a direct measurement of resistance to obtain the required sensor values, in order to minimize costs. Second, due to the non-directional nature of single odour measurements, a stereo nose architecture with two tubes or "nostrils" was used (see Fig. 2) to measure the spatial gradient of the gas concentration. This is the simplest architecture that can be used to measure the instantaneous gra- 
(a)

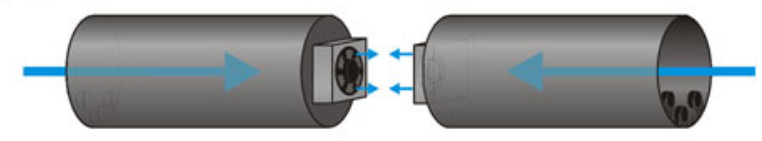

(b)

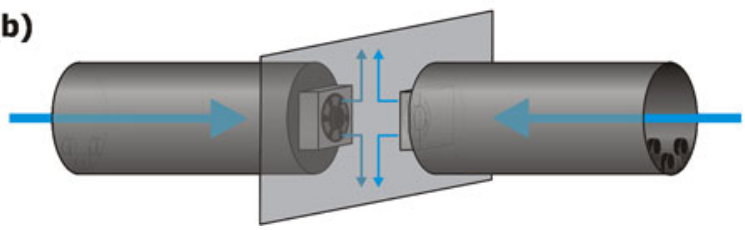

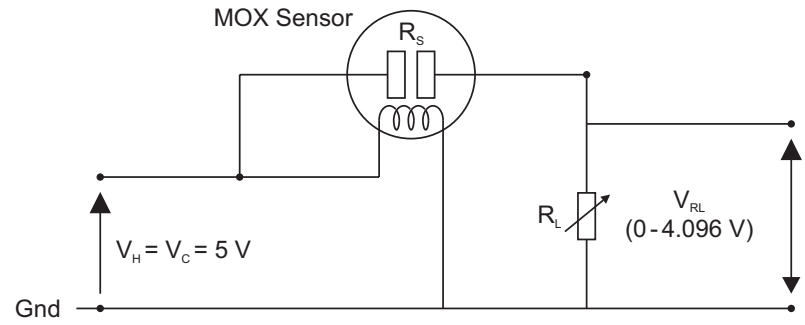

Figure 3: Electrical circuit used in the Mark III mobile nose to measure the resistance of the metal oxide gas sensors.

posed to a deoxidising gas, the potential barrier at the grain boundary is lowered, and thus the resistance of the surface layer decreases. In order to control the sensor's sensitivity, the semiconductor can be doped with different catalytic metal additives (e.g., Pt, Ir, Pd) or different semiconducters (tin dioxide, zinc oxide, etc.) can be used [2]. The selectivity of metal oxide sensors can be modified, however, only slightly in this way. Consequently these sensors have a fairly non-specific response in general. So, although the sensitivity of the Figaro sensors used in this work is optimised for a particular target gas, as indicated in Table 1, they all respond strongly to alcoholic substances, which were used to provide the gas source in our experiments. Even the TGS 2610 sensors, which are especially designed to have low sensitivity to alcohol vapours, supply a sufficiently high signal, even though it is lower than for the other sensors. Besides their low selectivity, metal oxide sensors

\begin{tabular}{|c|c|}
\hline Model & Typical substances detected \\
\hline TGS 2600 & hydrogen, carbon monoxide \\
\hline TGS 2610 & propane, butane \\
\hline TGS 2620 & organic solvents, carbon monoxide \\
\hline
\end{tabular}

Table 1: The Figaro gas sensors used in this work and their selectivity characteristics.

have other drawbacks, like their comparatively high power consumption (caused by the heating device) and weak durability. However, these sensors were chosen for the Mark III mobile nose because they are inexpensive, highly sensitive and relatively unaffected by changing environmental conditions like room temperature or humidity.

To obtain the signal output, the electrical circuit that is shown in Fig. 3 was used. A load resistor $R_{L}$ is connected in series with each gas sensor and the voltage across the load resistor $V_{R L}$ is measured. This value is then converted by a 10 bit $\mathrm{A} / \mathrm{D}$ converter that covers an input range of $0-$ $4.096 \mathrm{~V}$, and transferred to the host computer using a wireless serial connection. An adjustable load resistor $R_{L}(1-$ 


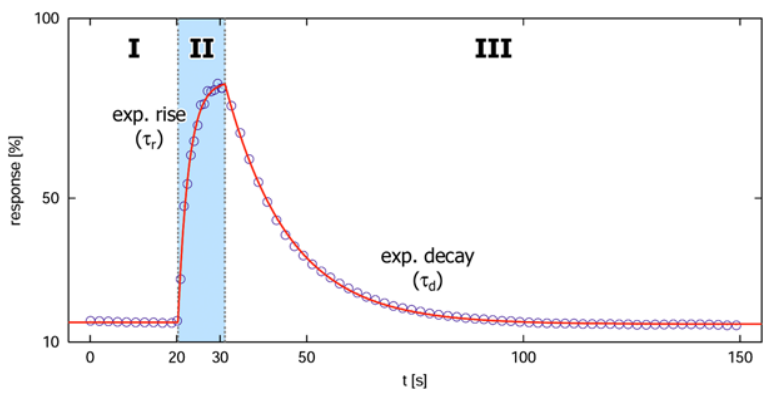

Figure 4: Real gas sensor readings (circles) together with the corresponding fitted dynamic response model (line). Relative conductance values are shown, recorded in an experiment where fans were used. Notice the three labelled regions of the applied model function (eqn. 1-5). Note also that 7 out of 8 readings were omitted in region I and III, and 2 out of 3 in region II for clarity of the plot.

$100 \mathrm{k} \Omega$ ) was used in order to be able to modify the mapped range according to the intended application.

In order to increase the spatial differentiation of the mobile nose, suction fans (Papst 405F) that generate an airflow of $8 \mathrm{~m}^{3} / \mathrm{h}$ were mounted inside the tubes. Thus, the exchange rate of air at the sensor's location is also increased and possible effects due to different driving speeds are reduced. As described below, with the addition of fans the recovery time could also be decreased.

\section{Dynamic Response Experiment}

In order to determine the dynamic response of the Mark III mobile nose as a whole, the following experiment was performed: alternately one set of sensors was exposed to a step stimulus, which was approximated by opening a bottle of ethanol in the direct vicinity of the sensors for a fixed period of time. For each of the 4 possible configurations that result from using or not using the fans and separating or not separating the two tubes with a septum, the following steps were repeated:

- wait for $20 \mathrm{~s}$,

- open a bottle of ethanol for $10 \mathrm{~s}$ at a distance of $\approx 1 \mathrm{~cm}$ alternately in front of the left- and right-side sensor set,

- close the bottle and wait for another $120 \mathrm{~s}$.

\section{Sensor Model}

The readings were analysed by fitting a sensor model to the values recorded during each trial. An example of a fitted model together with the corresponding gas sensor readings is shown in Fig. 4. Due to the complexity of the interaction between metal oxide sensors and their environment, no physically justified general description of this process is available. It is, however, sufficient for our concerns to assume an ideal first-order sensor and thus model the dynamic response to a step stimulus as an exponential rise and decay. To this end, the applied model (see eq. 1-5) separates into three parts according to the three regions shown in Fig. 4.

$$
\begin{gathered}
r(t)=\left\{\begin{array}{cl}
r_{I}(t) & \text { if } t<t_{S} \\
r_{I I}(t) & \text { if } t_{S}<t<t_{S}+\Delta t \\
r_{I I I}(t) & \text { if } t_{S}+\Delta t<t
\end{array}\right. \\
r_{I}(t)=R_{0} \\
r_{I I}(t)=R_{0}+\left(R_{\text {max }}-R_{0}\right)\left(1-\exp \left(\frac{-\left(t-t_{S}\right)}{\tau_{r}}\right)\right) \\
r_{I I I}(t)=R_{0}^{\prime}+\left(R_{\text {max }}^{*}-R_{0}^{\prime}\right) \exp \left(\frac{-\left(t-t_{S}-\Delta t\right)}{\tau_{d}}\right) \\
R_{\text {max }}^{*}=R_{0}+\left(R_{\max }-R_{0}\right)\left(1-\exp \left(\frac{-\Delta t}{\tau_{r}}\right)\right)
\end{gathered}
$$

The model contains 7 adjustable parameters: the response level before $\left(R_{0}\right)$ and after $\left(R_{0}^{\prime}\right)$ the stimulus, the saturation level $\left(R_{\max }\right)$, the time constants of rise $\left(\tau_{r}\right)$ and decay $\left(\tau_{d}\right)$, the time before the sensor started to respond $\left(t_{S}\right)$, and the duration of the rising period $(\Delta t)$. To determine these parameters, the model was fitted to 600 data points recorded per trial using the Marquardt-Levenberg algorithm [3].

\section{Results}

The fitting results for the 6 sensors used are summarised in Table 2. This includes the parameter that describes the average duration of the rising period, and the average of the time constants of rise and decay. Each of these values was calculated from the fit parameters for those trials where the stimulus was placed on the same side as the corresponding sensor. Twelve such trials (six on each side) were performed for each configuration except the one with fans and septum, which was used for the experiments described below. With this configuration a total of 18 trials was performed. A characteristic response to two consecutive step stimuli is shown in Fig. 5 for each configuration tested.

The values listed in Table 2 were calculated as a weighted average and standard deviation respectively, with the weights being the quadratic inverse asymptotic standard error of the corresponding fit parameter. The calculated 
(a)

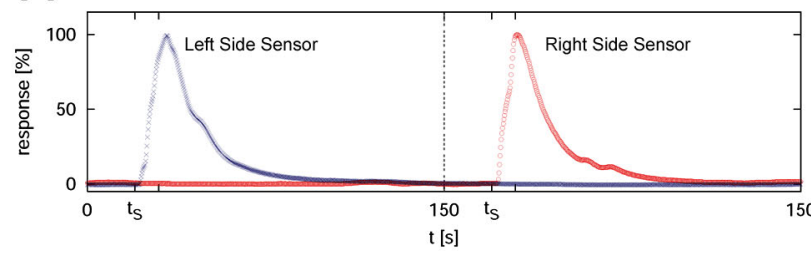

(c)

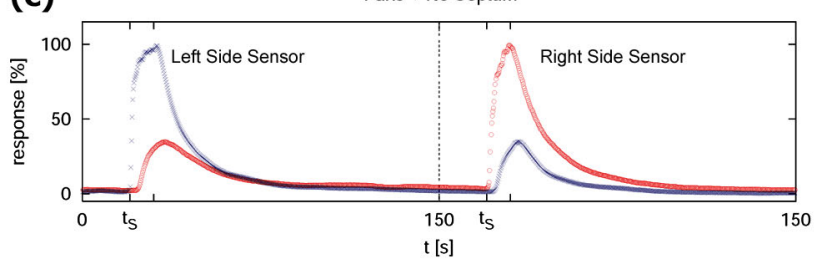

(b)

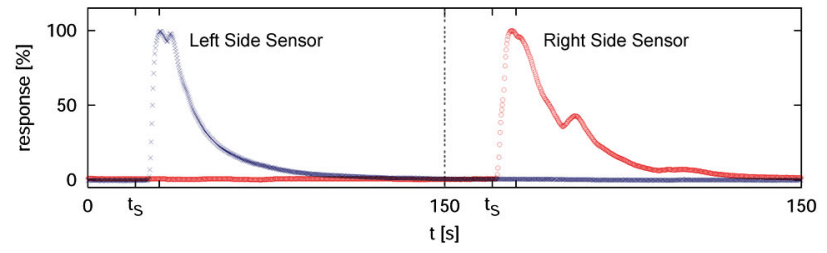

(d)

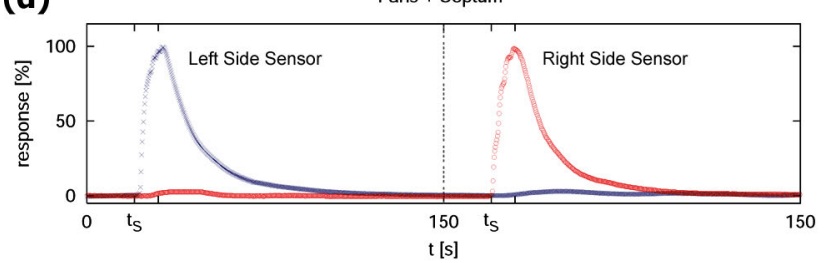

Figure 5: Dynamic response of two opposite TGS 2600 sensors to two consecutive step stimuli on alternate sides of the robot.

mean values are not guaranteed to be the best overall estimate for non-linear fit parameters. On the other hand, extracting these parameters by fitting to all the recorded runs simultaneously is not possible in a straightforward way due to the variations in experimental conditions. Consequently, the weighted mean provides a reasonably precise insight into the characteristics of our mobile nose.

The last column in Table 2 contains the difference between the response time $t_{S}$ of the sensor on the side on which the stimulus was generated, and the response time of the sensor's counterpart on the other side. When the fans remained switched off, the remote sensor's response was too weak to obtain a meaningful fit. Therefore the last column contains values only for those experiments where fans were used.

Analysing the values in Table 2 shows firstly that fitting with the assumed model is able to reproduce the stimulus' duration. The agreement with the intended time of $10 \mathrm{sec}-$ onds is quite good, considering the fact that the actual effective duration depends on varying factors such as the local airflow or how the bottle was opened and closed. The obvious outliers for the TGS 2620 sensors (indicated by a large standard deviation) in the configuration without fans and a septum were caused by the fact that in all the corresponding trials the response declined extremely slowly and unsteadily. Thus, the applied fitting algorithm was not able to ascertain the first clear drop at the end of the rising period II.

Next, it is interesting to compare results of measurements where fans were used with those where the fans remained switched off. Checking the rising time constants $\overline{\tau_{r}}$ using a paired Student's t-test [3] shows no statistically significant difference with respect to the usage of fans $\left(p_{H_{0}}=0.9960\right)$. This also holds if no normal distributed observations were assumed by means of a distribution-free Wilcoxon matched-pairs signed-rank test $\left(p_{H_{0}}=0.9697\right)$.

By contrast, the decay time constants $\overline{\tau_{d}}$ turn out to be clearly higher in the case where no fans were used. The opposite hypothesis can be rejected with high statistical significance (paired Student's t-test: $p_{H_{0}}=0.0015$, Wilcoxon matched-pairs signed-rank test: $p_{H_{0}}=0.0005$ ).

A reasonable estimate of the sensor response is provided by the mean values of the time constants (averaged over all six sensors):

$$
\begin{gathered}
\tau_{r} \approx 1.8 s \\
\tau_{d}^{(\text {NoFans })} \approx 20.7 s \quad \tau_{d}^{(\text {Fans })} \approx 11.1 s
\end{gathered}
$$

Note that the rise and decay constants depend generally on the sensor type. In addition, these characteristics vary between different sensors of the same type, and also for one sensor over prolonged periods of time. Finally, they also depend on the gas concentration. Bearing these restrictions in mind, the approximation given in (6) and (7) provides a reasonable notion of the mobile nose's characteristics: the use of fans does not influence the response time to a presented stimulus, but rather lowers the time needed for the sensors to recover after the stimulus has been removed. This is caused by the higher rate of air exchange effected by the fans.

On the other hand, an increased exchange of gas provokes also a less clear distinction of the measured response with respect to the sensors location. Although the air streams caused by the fans are directed against each other (see Fig. 2), a considerable amount of gas is transported from one side to the other if no solid separation is used inbetween the suction tubes. This is indicated by the relative strength of the response, as can be seen in Fig. 5 (c), and by the delay of the response time $t_{S}$. Again a significant dif- 


\begin{tabular}{|cc|cc|cc|ccc|}
\hline \multirow{2}{*}{ Config. } & \multirow{2}{*}{ Type } & \multicolumn{2}{|c|}{$\overline{\Delta t}[\mathrm{~s}]$} & \multicolumn{2}{c|}{$\overline{\tau_{r}}[\mathrm{~s}]$} & \multicolumn{2}{c|}{$\overline{\tau_{d}}[\mathrm{~s}]$} & $\overline{|c|}$ \\
& & Left & Right & Left & Right & Left & Right & \\
\hline No Fans & TGS 2600 & $11.04 \pm 1.85$ & $9.09 \pm 1.69$ & $2.27 \pm 0.87$ & $1.40 \pm 1.37$ & $15.55 \pm 1.28$ & $15.22 \pm 3.34$ & - \\
$\&$ & TGS 2610 & $12.63 \pm 1.94$ & $7.68 \pm 2.80$ & $0.81 \pm 0.65$ & $0.33 \pm 0.44$ & $19.55 \pm 3.00$ & $13.02 \pm 2.27$ & - \\
No Septum & TGS 2620 & $12.35 \pm 2.25$ & $8.15 \pm 2.05$ & $1.47 \pm 1.49$ & $2.39 \pm 0.82$ & $29.25 \pm 4.69$ & $28.51 \pm 7.33$ & - \\
\hline No Fans & TGS 2600 & $8.43 \pm 3.06$ & $7.06 \pm 3.67$ & $1.99 \pm 0.77$ & $0.99 \pm 0.48$ & $15.30 \pm 2.23$ & $20.68 \pm 4.95$ & - \\
$\&$ & TGS 2610 & $8.78 \pm 3.12$ & $7.46 \pm 1.59$ & $2.77 \pm 1.31$ & $0.56 \pm 0.28$ & $16.57 \pm 5.53$ & $18.17 \pm 5.51$ & - \\
Septum & TGS 2620 & $14.21 \pm 14.15$ & $23.39 \pm 13.67$ & $4.10 \pm 1.33$ & $2.05 \pm 0.56$ & $19.82 \pm 6.37$ & $36.74 \pm 4.63$ & - \\
\hline Fans & TGS 2600 & $11.16 \pm 1.17$ & $10.45 \pm 0.56$ & $2.13 \pm 0.76$ & $1.86 \pm 0.83$ & $12.73 \pm 1.05$ & $14.98 \pm 1.38$ & $3.40 \pm 0.75$ \\
$\&$ & TGS 2610 & $10.23 \pm 0.70$ & $9.86 \pm 0.51$ & $2.53 \pm 1.06$ & $1.06 \pm 1.11$ & $9.22 \pm 1.25$ & $9.64 \pm 2.76$ & $3.18 \pm 0.43$ \\
No Septum & TGS 2620 & $10.36 \pm 0.55$ & $10.59 \pm 0.46$ & $2.26 \pm 0.96$ & $1.44 \pm 0.36$ & $7.86 \pm 0.94$ & $12.53 \pm 0.54$ & $3.94 \pm 1.23$ \\
\hline Fans & TGS 2600 & $9.81 \pm 1.81$ & $10.39 \pm 0.82$ & $0.29 \pm 0.43$ & $2.27 \pm 0.35$ & $12.92 \pm 1.28$ & $13.63 \pm 0.64$ & $6.28 \pm 2.87$ \\
$\&$ & TGS 2610 & $9.66 \pm 1.05$ & $9.51 \pm 1.00$ & $2.24 \pm 0.85$ & $1.26 \pm 0.63$ & $8.82 \pm 0.87$ & $10.89 \pm 3.08$ & $5.49 \pm 3.21$ \\
Septum & TGS 2620 & $7.94 \pm 3.59$ & $9.99 \pm 0.58$ & $2.29 \pm 1.22$ & $1.52 \pm 0.63$ & $9.30 \pm 2.70$ & $10.49 \pm 1.46$ & $5.96 \pm 3.98$ \\
\hline
\end{tabular}

Table 2: Summarised fitting results of the dynamic sensor response to a step stimulus. The average and standard deviation of the rising period $\Delta t$, the time constants of rise $\tau_{r}$ and decay $\tau_{d}$ as well as the delay in responding $\Delta t_{S}$ is given for each sensor.

ference could be assessed by testing on the equality of the calculated time difference $\overline{\Delta t_{S}}$, at least if a normal distribution is assumed (paired Student's t-test: $p_{H_{0}}=0.011$ ). This result may be overly optimistic due to the small amount of compared observations (Wilcoxon matched-pairs signedrank test gives $p_{H_{0}}=0.25$ ). Nevertheless the implication of the described experiment is apparent: in order to get measurements that reflect the instantaneously sensed concentration as closely as possible, the usage of fans is clearly favourable. On the other hand, the airstream produced by the fans must then be separated carefully as it is done, for example, in the Mark III mobile nose by using a septum.

\section{Applications}

The Mark III mobile nose has been used successfully in several applications. Two of them are briefly summarised below.

\subsection{Reactive Gas Source Localisation}

This work investigated the suitability of reactive localisation strategies (based on the instantaneously measured spatial concentration gradient) to localise a static odour source in an unstructured indoor environment. In particular, the condition of an environment that is not artificially ventilated to produce a strong unidirectional airflow is considered. The propagation of the analyte molecules is therefore dominated by turbulence and convection flow rather than diffusion [4], thus creating a patchy distribution of spatially distributed eddies.

Two alternative strategies were implemented utilising a direct sensor-motor coupling (essentially a Braitenberg ve- hicle [5]). Both were shown to be useful for localisation. With uncrossed inhibitory connections (where the robot tries to turn towards increased concentrations) the average path length the robot needs to move to the source is reduced by a factor of two compared to random search.

However, an additional mechanism to detect that the gas source has been found is needed for real world applications because the location of the source does not usually correspond to the global concentration maximum.

A possibility to solve the problem of declaring that the gas source has been found is provided by using crossed connections. Here, the robot evades each local concentration maximum, including the one that is caused by the source. These experiments showed that a gas source can be detected by exploiting the fact that local concentration maxima occur more frequently near the odour source compared to distant regions.

A detailed description of these experiments is given in [6].

\subsection{Gas Concentration Mapping}

A new technique for modelling gas distributions by creating concentration gridmaps was presented in [7]. By contrast to metric gridmaps extracted from sonar or laser range scans, a single measurement from a mobile nose provides information about a comparatively small area.

To overcome this problem the mapping technique introduced uses a Gaussian density function to model the decreasing likelihood that a particular reading represents the true concentration with respect to the distance from the point of measurement.

Using data recorded with the Mark III mobile nose, the 

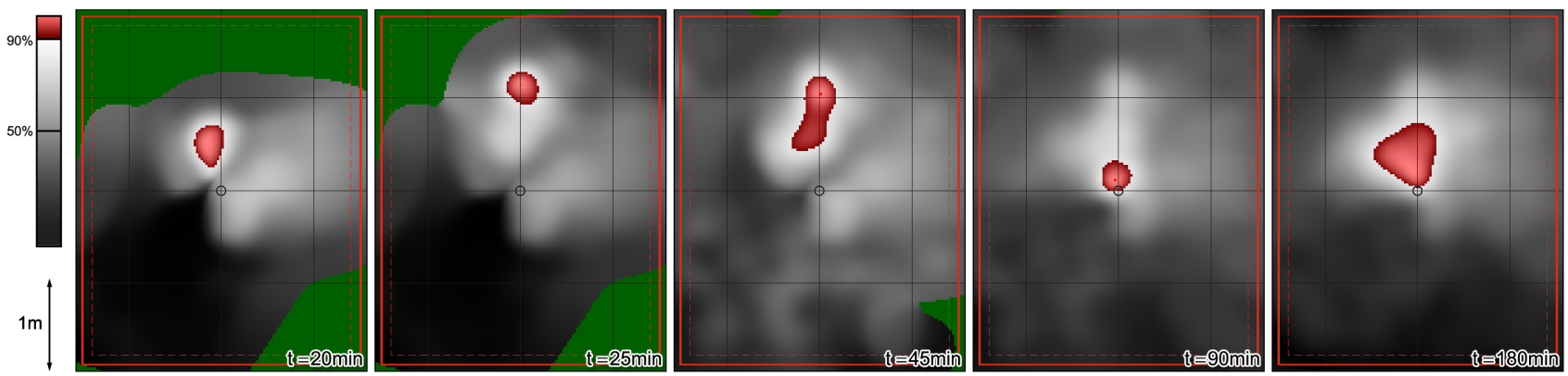

Figure 6: Time evolution of a concentration gridmap recorded while a reactive localisation strategy was applied. The gas source was located in the middle of the searched area. It is indicated by a circle in the concentration maps shown above.

suitability of this method regarding the slow response and recovery of the sensors could be demonstrated. An example of the time evolution of a concentration gridmap is given in Fig. 6.

It could be further shown that time-invariant structures are represented more quickly in the mapping process using reactive control strategies that increase the time the robot spends in regions of high concentration.

Finally, the suitability of the introduced concentration mapping technique for gas source localisation was evaluated. Although the maximum of the mapped concentration does not necessarily correspond to the location of the gas source, it often provides a reasonably good estimate that allows localisation of the gas source with an accuracy of less than $1 \mathrm{~m}$.

A detailed description of the corresponding experiments is given in [7].

\section{Conclusions}

The statistical analysis of the experiments provided an estimate of the exponential time constants of rise and decay of the Mark III mobile nose. While the metal oxide gas sensors respond fairly quickly to an increased concentration, they recover much more slowly, resulting in a considerable integration of successive measurements.

As indicated by the approximated values given in (6) and (7), the time constant of rise is almost unaffected no matter whether fans were used or not. By contrast, the time constant of decay is almost half as long if fans were used, indicating that it is favourable to use fans in order to get measurements that reflect the instantaneously sensed concentration as closely as possible. On the other hand, the airstream produced by the fans must then be separated carefully, for example, by using a septum or dividing wall.

The Mark III mobile nose has been utilised successfully in a number of experiments, including reactive gas source localization with a smelling Braitenberg vehicle [6], localization by concentration peak avoidance [6], and gas distribution mapping [7].

\section{References}

[1] Julian W. Gardner and Philip N. Bartlett. Electronic Noses - Principles and Applications. Oxford Science Publications, Oxford, 1999.

[2] Toyosaka Moriizumi. Biomimetic Sensing Systems With Arrayed Nonspecific Sensors. In Proceedings of the 8th International Conference on Solid-State Sensors and Actuators (Transducers 95), pages 39-42, 1995.

[3] William H. Press, Brian P. Flannery, Saul A. Teukolsky, and William T. Vetterling. Numerical Recipes: The Art of Scientific Computing. Cambridge University Press, Cambridge (UK) and New York, 2nd edition, 1992.

[4] Michael R. Wandel, Achim Lilienthal, Tom Duckett, Udo Weimar, and Andreas Zell. Gas Distribution in Unventilated Indoor Environments Inspected by a Mobile Robot. In Proceedings of the IEEE International Conference on Advanced Robotics (ICAR 2003), Coimbra, Portugal, 2003.

[5] Valentino Braitenberg. Vehicles: Experiments in Synthetic Psychology. MIT Press/Bradford Books, 1984.

[6] Achim Lilienthal and Tom Duckett. Experimental Analysis of Smelling Braitenberg Vehicles. In Proceedings of the IEEE International Conference on Advanced Robotics (ICAR 2003), Coimbra, Portugal, 2003.

[7] Achim Lilienthal and Tom Duckett. Gas Source Localisation by Constructing Concentration Gridmaps with a Mobile Robot. In Proceedings of the European Conference on Mobile Robots (ECMR 2003), Warsawa, Poland, 2003. 